\title{
Peculiaridade dos Pacientes com Arritmias Hereditárias na Pandemia pela COVID-19
}

\author{
Peculiar Aspects of Patients with Inherited Arrhythmias during the COVID-19 Pandemic
}

Luciana Sacilotto, ${ }^{10}$ Natalia Quintella Sangiorgi Olivetti, ${ }^{1}$ Cristiano Faria Pisani, ${ }^{1}$ Tan Chen Wu, ${ }^{10}$ Ludhmila Abrahão Hajjar, ${ }^{10}$ Sissy Lara de Melo, ${ }^{10}$ Sávia Christina Pereira Bueno, ${ }^{1}$ Esteban Wisnivesky Rocca Rivarola, ${ }^{1}$ Muhieddine Omar Chokr, ${ }^{1}$ (1) Carina Abigail Hardy, ${ }^{1}$ Denise Tessariol Hachul, ${ }^{1}$ Francisco Carlos da Costa Darrieux, ${ }^{1}$ Mauricio Ibrahim Scanavacca ${ }^{1}$

Instituto do Coração do Hospital das Clínicas da Faculdade de Medicina da Universidade de São Paulo, ${ }^{1}$ São Paulo, SP - Brasil

\section{Resumo}

Desde dezembro de 2019, observamos o rápido avanço da síndrome respiratória aguda grave causada pelo coronavírus 2019 (SARS-CoV-2). O impacto da evolução clínica de uma infecção respiratória é pouco conhecido em pacientes portadores de arritmias hereditárias, devido à baixa prevalência dessas doenças. Os pacientes que apresentam quadros infecciosos podem exacerbar arritmias primárias ocultas ou bem controladas, por diversos fatores, tais como febre, distúrbios eletrolíticos, interações medicamentosas, estresse adrenérgico e, eventualmente, o próprio dano miocárdico do paciente séptico. O objetivo desta revisão é destacar os principais desafios que podemos encontrar durante a pandemia pela Covid 19, especificamente nos pacientes com arritmias hereditárias, com destaque para a síndrome do QT longo congênito (SQTL), a síndrome de Brugada (SBr), a taquicardia ventricular polimórfica catecolaminérgica (TVPC) e a cardiomiopatia arritmogênica do ventrículo direito.

Desde dezembro de 2019, observamos o rápido avanço da síndrome respiratória aguda grave causada pelo novo coronavírus (SARS-CoV-2), que registrou os primeiros casos em Wuhan, na China, e atingiu o Brasil recentemente. Estudos retrospectivos demonstraram que a idade avançada foi um preditor de mortalidade independente na Covid-19. Os demais fatores de risco, com impacto na mortalidade, foram hipertensão arterial sistêmica, doença pulmonar obstrutiva crônica, imunossupressão, diabetes melito tipo 2, obesidade e cardiopatia severa (insuficiência cardíaca, doença coronária ou miocardiopatias). ${ }^{1,2}$

De forma geral, as complicações por arritmias em pacientes com pneumonia são relativamente comuns, destacando-se a fibrilação atrial. ${ }^{3,4}$ A parada cardíaca ocorre em cerca de 3\% dos pacientes internados, ${ }^{5}$ porém menos de $20 \%$ dos eventos

\section{Palavras-chave}

COVID-19; Cardiomiopatia Arritmogênica do Ventrículo Direito, Síndrome de Brugada; Síndrome do QT Longo; Taquicardia Ventricular Polimórfica Catecolaminérgica.

Correspondência: Mauricio Ibrahim Scanavacca •

Instituto do Coração - Unidade de Arritmia - Av. Dr. Enéas de Carvalho

Aguiar, 44. CEP 05403-900, São Paulo, SP - Brasil

E-mail: mauricio.scanavacca@incor.usp.br

Artigo recebido em 05/05/2020, revisado em 11/11/2020, aceito em $25 / 11 / 2020$

DOI: https://doi.org/10.36660/abc.20200391 hospitalares são descritos como ritmo passível de reversão elétrica (cardioversão ou desfibrilação), ou seja, taquicardia/ fibrilação ventricular (TV/FV). ${ }^{6}$ Nesses pacientes, o principal mecanismo arritmogênico é o dano miocárdico por isquemia ou inflamação. ${ }^{4}$

O impacto da evolução clínica da sepse, ou particularmente das infecções respiratórias, é pouco conhecido em pacientes portadores de arritmias hereditárias, devido à baixa prevalência dessas doenças. ${ }^{7}$ Além disso, em sua maioria, as arritmias hereditárias apresentam, em geral, penetrância incompleta e idade dependente, ${ }^{8}$ sendo mais expressas em pacientes jovens que, por sua vez, apresentam risco menor de desenvolver quadros infecciosos graves.

Os pacientes que apresentam quadros infecciosos mais graves podem exacerbar arritmias ocultas ou bem controladas, por diversos fatores, tais como febre, distúrbios eletrolíticos, interações medicamentosas, estresse adrenérgico e, eventualmente, o próprio dano miocárdico do paciente séptico. Todos esses fatores podem alterar o equilíbrio dos canais iônicos, tornando os pacientes com arritmias hereditárias potencialmente mais vulneráveis.

Os eventos fatais em pacientes com arritmias hereditárias podem ser desencadeados por estresse físico e emocional. Os impactos psicossociais da pandemia relacionados a depressão, estresse, ansiedade e síndrome do pânico, agravados pelo isolamento social, enfrentamento do medo e do luto, podem predispor a maior ocorrência de arritmias. ${ }^{9}$ A eventual necessidade de suspensão temporária das medicações (betabloqueadores e antiarrítmicos) em pacientes com instabilidade hemodinâmica, uso de fármacos vasopressores com efeito catecolaminérgico e distúrbios hidroeletrolíticos pode estar associada a maior risco de eventos potencialmente fatais. Portanto, o período da pandemia, por si só, já alerta para a necessidade de garantir vigilância e orientações direcionadas para esses pacientes, que, por serem portadores de doenças raras, não serão bem representadas em estudos clínicos. Em caso de infecção pelo SARS-COV2, não há dados epidemiológicos suficientes nessa população para categorizar os pacientes em grupo de risco, gerando insegurança para o médico e para o paciente. Na Tabela 1, elencamos os cuidados gerais intra e extra-hospitalares que podem ser adotados nos pacientes com arritmias genéticas previamente conhecidas.

Assim, é importante revisar os principais desafios que podemos encontrar durante a pandemia da Covid 19, 7 especificamente nessa subpopulação, com destaque para a síndrome do QT longo congênito (SQTL), a síndrome 
Tabela 1 - Cuidados sugeridos durante a pandemia de Covid-19 em pacientes com arritmias hereditárias

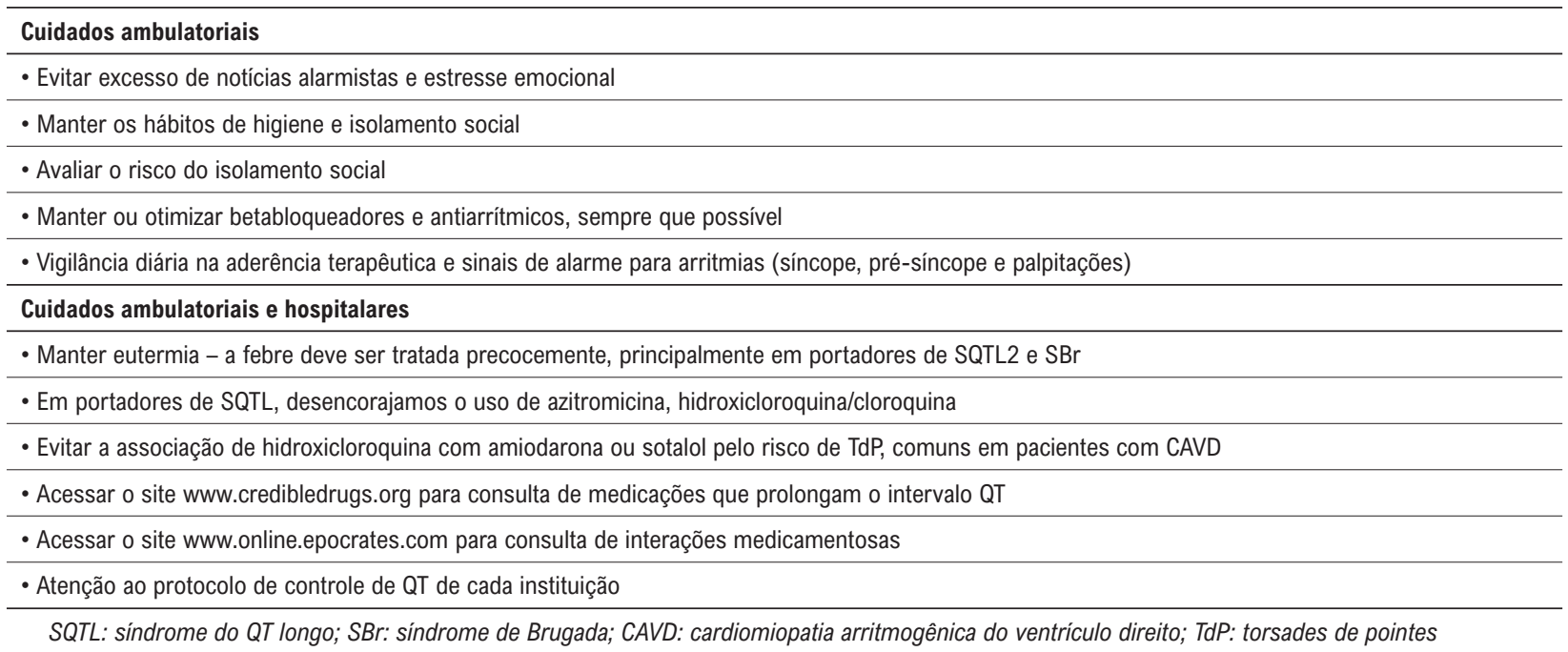

de Brugada $(\mathrm{SBr})$, a taquicardia ventricular polimórfica catecolaminérgica (TVPC) e a cardiomiopatia arritmogênica do ventrículo direito (CAVD).

\section{Síndrome do QT longo congênito}

\section{Aspectos gerais}

A SQTL ocorre em 1 em 2.000 pessoas, é caracterizada pelo prolongamento do intervalo QT no eletrocardiograma (ECG) de repouso de 12 derivações e uma propensão para síncope ou convulsões por torsades de pointes (TdP) e morte súbita cardíaca (MSC). ${ }^{10}$

Está associada a defeitos que acentuem as correntes despolarizantes de sódio e cálcio (INa e ICa L, respectivamente) ou atenuem correntes repolarizantes de potássio (IKs, IKr e IK1), levando a um prolongamento no potencial de ação cardíaco, refletindo no intervalo QT prolongado. ${ }^{11}$ Há o reconhecimento de peculiaridades clínicas, que permitem classificar a SQTL em subtipos, principalmente SQTL 1 (canal IKs, gene KCNQ1), 2 (canais hERG ou IKr, gene $K C N H 2$ ) e 3 (canal INa, gene SCN5A).

A medida do intervalo QT deve ser preferencialmente realizada nas derivações DII ou V5 (Figura 1), corrigida pelo intervalo RR precedente, utilizando a fórmula de Bazzet, preferencialmente em frequência cardíaca entre 60 a $80 \mathrm{bpm} .{ }^{12}$ As diretrizes atuais definem um valor prolongado de QTc quando acima e $450 \mathrm{~ms}$ em homens e $460 \mathrm{~ms}$ em mulheres. No entanto, 5\% a 10\% dos indivíduos saudáveis têm um QTC > 460ms, sendo necessários outros dados clínicos para compor o diagnóstico de SQTL. Nesses casos, recomenda-se a utilização do escore de Schwartz. ${ }^{13,14}$ Apenas quando os valores de QTc são maiores que 480ms, na ausência de causas secundárias, há maior especificidade para a SQTL. ${ }^{14}$ Por outro lado, cerca de 30\% dos pacientes têm a forma oculta da SQTL, representada por alteração genética e intervalo QT normal, sendo o histórico familiar ou o teste genético relevantes para suspeita clínica nesses pacientes.

A conduta padrão em paciente com SQTL é o bloqueio beta-adrenérgico, com uso de nadolol/propranolol, além de evitar medicações que prolonguem o intervalo QT. A indicação de denervação simpática cardíaca esquerda e do cardiodesfibrilador implantável (CDI) permanece reservada nos casos de maior risco de eventos arrítmicos potencialmente fatais. ${ }^{14}$

\section{Cuidados na pandemia pela Covid-19}

Os canais iônicos cardíacos são modulados pelo sistema nervoso autônomo, pois o tempo de repolarização cardíaca é constantemente ajustado pela frequência cardíaca. Em situações de estresse adrenérgico, temos aumento da frequência cardíaca, com fosforilação dos canais de potássio e aumento da sua velocidade de abertura em condições normais. Em situações de defeitos proteicos geneticamente determinados, esse ajuste é prejudicado, lentificando a negativação do potencial de membrana, o que permite a entrada desequilibrada de cálcio e ocorrência de póspotenciais precoces. Enquanto a frequência cardíaca permanece elevada, pode haver alguma inibição desses potenciais, entretanto quando a situação metabólica leva a bradicardia ou irregularidade do intervalo RR, observamos um aumento da dispersão da repolarização e maior ocorrência de pós-potenciais de fase 3. Dependendo do limiar de excitabilidade da célula, os pós-potenciais geram extrassístoles ventriculares, $\mathrm{TdP} / \mathrm{FV}$. $^{11}$

A suspensão ou redução de betabloqueadores usados cronicamente no tratamento da SQTL pode agravar a ocorrência de arritmias potencialmente fatais. Portanto, as medicações devem ser rigorosamente mantidas em pacientes em tratamento domiciliar e, no doente crítico, a decisão deve ser pautada pela estabilidade hemodinâmica. 


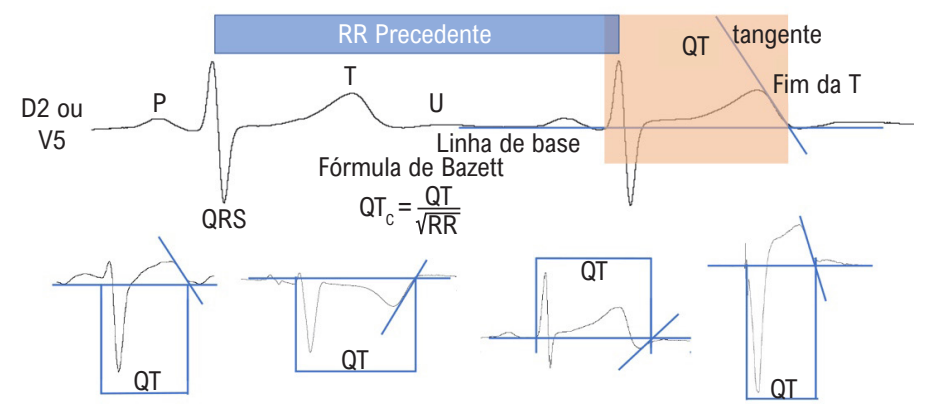

Figura 1 - Medida do intervalo QT em paciente com síndrome do QT longo. Fonte: acervo InCor-HC-FMUSP.

O equilíbrio das correntes iônicas depende do nível celular desses íons, que pode ser dinâmico em doentes críticos. A hipocalemia, a hipocalcemia e a hipomagnesemia provocam pós-potenciais precoces, aumentam a dispersão da repolarização e levam a TdP com mais facilidade em portadores de SQTL. A manutenção do potássio entre 4,5 a $5 \mathrm{mg} / \mathrm{dL}$ pode ser uma estratégia protetora. ${ }^{7}$

A sepse ou o choque séptico são estados adrenérgicos por dor/desconfortos, além do próprio estado inflamatório. Os portadores de SQTL 1 e SQTL 2 são os que apresentam arritmias desencadeadas principalmente por estresse adrenérgico e, portanto, mais vulneráveis nessas situações críticas. A presença da febre pode influenciar as propriedades biofísicas dos canais sensíveis à temperatura, particularmente os canais hERG, acometidos na SQTL2. ${ }^{15}$ Por outro lado, a hipotermia também está associada ao prolongamento do intervalo QT, porém com baixo risco de indução de TdP. ${ }^{16}$

As lesões miocárdicas inflamatórias alteram o potencial de membrana celular, gerando dispersão de repolarização e suscetibilidade às arritmias ventriculares. ${ }^{17}$ Além disso, citocinas e autoanticorpos podem se ligar aos canais iônicos cardíacos levando à canalopatia induzida pela inflamação, com maior gravidade presumida em pacientes com SQTL. ${ }^{18}$

Os fármacos que bloqueiam os canais de potássio (hERG) podem prolongar ainda mais a repolarização cardíaca, aumentando o risco de arritmias fatais. A indicação dessas medicações deve ser rigorosamente ponderada, principalmente em regime extra-hospitalar, pela falta de monitoramento contínuo. Além disso, os medicamentos que levam à inibição do citocromo P450 3A4 (CYP3A4) podem aumentar ainda mais o nível plasmático de fármacos que prolongam o intervalo QT. ${ }^{7}$

A lista de medicações está disponível em www.crediblemeds. org e inclui a cloroquina/hidroxicloroquina e a azitromicina. No doente crítico, outras medicações de risco são frequentemente administradas, tais como antieméticos (ondasentrona e metoclopramida), ${ }^{19}$ antipsicóticos (haloperidol), fármacos vasoativos (noradrenalina, dobutamina), analgésicos (tramadol) e sedativos (etomidato, propofol). ${ }^{20}$
A cloroquina e a hidroxicloroquina, associadas ou não à azitromicina, apresentam uso controverso em pacientes com Covid-19, sendo sua eficácia demonstrada in-vitro, ${ }^{21}$ porém ainda sem estudos clínicos comprobatórios. Em recente publicação, Mazzanti et al. sugerem que uma dose cumulativa de hidroxicloroquina de $2 \mathrm{~g}$ em 5 dias, conforme adotada em $30 \%$ de todos os estudos em andamento com hidroxicloroquina (www.clinicaltrials.gov), leva a um prolongamento modesto do intervalo QTc em pacientes com QTc basal normal (média de aumento de 20ms) e sem aumento do risco de complicações arrítmicas ameaçadoras à vida. ${ }^{22} \mathrm{Em}$ outra série de pacientes com lúpus eritematoso sistêmico, com intervalo QTc médio de $443 \pm 25,3 \mathrm{~ms}(373-518 \mathrm{~ms})$, houve um prolongamento do intervalo QTc em 14,2\% dos pacientes em uso de cloroquina. ${ }^{23}$ Considerando que os pacientes com SQTL já apresentam maior suscetibilidade a pró-arritmias tipo $T d P$, o uso da cloroquina ou hidroxicloroquina, principalmente quando associado à azitromicina, deve ser desencorajado em pacientes com SQTL. ${ }^{12}$

A polifarmácia é um item que exige precaução multidisciplinar, por parte de médicos e farmacêuticos; em pacientes com SQTL, isso se torna uma preocupação ainda mais relevante pelo risco iminente de morte súbita se expostos a tais fármacos. Especificamente nesses pacientes, devemos discutir pontualmente riscos e benefícios de cada medicação.

Em caso de ocorrência de TdP com degeneração para FV, há necessidade de desfibrilação e medidas de reanimação cardiopulmonar. O TdP costuma ter uma apresentação autolimitada com resolução espontânea; entretanto, o mais desafiador é a prevenção da recorrência do $T d P$. As medidas urgenciais incluem minimizar medicações pró-arrítmicas e suprimir os fatores que geram pós-potenciais precoces. ${ }^{24}$

A principal medida para a supressão dos pós-potenciais precoces na SQTL é evitar bradicardia e "pausa-dependência". Em pacientes com SQTL adquirida, é possível tentar medidas farmacológicas, como isoprenalina endovenosa; por outro lado, em pacientes com SQTL congênita, o overdriving deve ser realizado apenas por meio de estímulo atrial ou ventricular, com marca-passo provisório (transcutâneo ou transvenoso). 


\section{Artigo de Revisão}

A administração de $2 \mathrm{~g}$ sulfato de magnésio, seguida de infusão contínua (3 a 10mg/min) é terapia coadjuvante em ambas as circunstâncias, com objetivo de reduzir a amplitude da oscilação do potencial de membrana na fase 3 do potencial de ação. Em caso de refratariedade, a sedação para cessar o estímulo adrenérgico pode ser necessária. ${ }^{25}$

\section{Síndrome de Brugada}

\section{Aspectos gerais}

A SBr acomete cerca de 1 em 5.000 pessoas, com predomínio no sexo masculino. O diagnóstico da $\mathrm{SBr}$ é definido pelo ECG, na presença de supradesnivelamento do segmento ST de $2 \mathrm{~mm}$, em pelo menos uma derivação precordial direita (V1-V2), em posição padrão (quarto espaço intercostal [EIC]) ou em EIC superiores (segundo ou terceiro EIC) (Figura 2), seguido de onda T negativa (padrão tipo 1). O principal desafio no diagnóstico e na classificação é que esse padrão eletrocardiográfico é dinâmico na maioria dos pacientes; ${ }^{14}$ portanto, pode ser documentado espontaneamente ou necessitar de teste provocativo, sob uso de fármacos específicos (p. ex., ajmalina).

Na ausência de causas secundárias, a presença do padrão eletrocardiográfico espontâneo de $\mathrm{SBr}$ tipo 1 é suficiente para o diagnóstico. Nos casos de $\mathrm{SBr}$ induzida por febre ou por testes provocativos, haveria a necessidade de adicionar dados clínicos pessoais e familiares para definir o diagnóstico com precisão. Recentemente, foi proposto um sistema de pontuação na SBr (escore de Shanghai), que pode ser utilizado como ferramenta diagnóstica. ${ }^{26}$

As causas secundárias, chamadas de fenocópias de SBr, incluem uso de fármacos que induzem ao supradesnivelamento do segmento ST (p. ex., tricíclicos), distúrbios eletrolíticos, isquemia miocárdica e outras formas de distorção do segmento ST (presença de pectus excavatum) (Tabela 2). ${ }^{27}$
A genética da Sbr é mais complexa do que as outras síndromes elétricas primárias. O SCN5A, primeiro gene identificado, ainda permanece como gene causal, mas todos os demais 20 genes citados na literatura recente carecem de correlação genótipo-fenótipo. ${ }^{28}$

$\mathrm{O}$ tratamento para os pacientes com $\mathrm{SBr}$ envolve evitar situações que facilitem a ocorrência das arritmias potencialmente fatais (TV/FV), como febre, uso de drogas ilícitas, libação alcoólica, refeições copiosas ou medicações que aumentem o bloqueio dos canais de sódio. A quinidina, importante bloqueador dos canais Ito, aparenta ser segura e reduz eventos arrítmicos no seguimento clínico de pacientes com alto risco.

A indicação CDI é reservada para pacientes que ou apresentaram TV sustentada espontânea documentada, parada cardiorrespiratória (PCR) recuperada ou, na prevenção primária, naqueles que apresentam maior risco de eventos arrítmicos, como na presença da síncope. O estudo eletrofisiológico (EEF) pode ser usado para estratificação dos pacientes assintomáticos, com resultados controversos. ${ }^{14} \mathrm{~A}$ ablação por radiofrequência do substrato da Sbr emergiu como tratamento coadjuvante para arritmias ventriculares recorrentes e está sendo estudada em pacientes sem eventos arrítmicos prévios. ${ }^{29}$

\section{Cuidados na pandemia pela Covid-19}

$\mathrm{O}$ primeiro aspecto em pacientes com $\mathrm{SBr}$ é a precisão diagnóstica, por se tratar de uma alteração eletrocardiográfica inespecífica, com vieses de interpretação. A lista de doenças que podem mimetizar a alteração eletrocardiográfica da SBr, chamadas de fenocópias, deve ser cuidadosamente analisada, para adequada orientação (ver Tabela 2). Podemos notar que várias fenocópias podem ocorrer no contexto infeccioso, como as miocardites, os distúrbios eletrolíticos, o tromboembolismo pulmonar e o infarto do miocárdico.

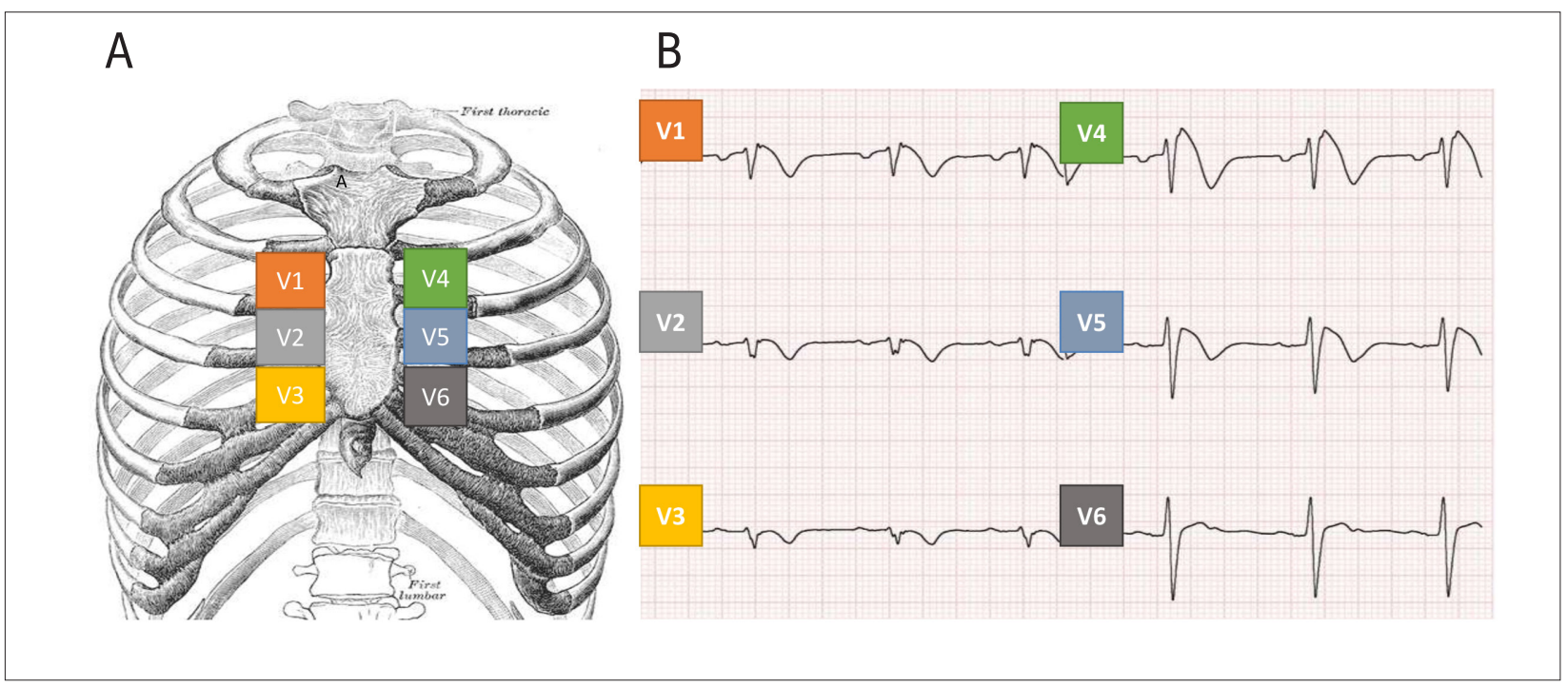

Figura 2 - Posicionamento dos eletrodos precordiais na síndrome de Brugada. A. Posicionamento das derivações precordiais para se obter maior sensibilidade na detecção do padrão típico de Brugada, demonstrado no eletrocardiograma (ECG) em B, com típico supradesnivelamento do segmento ST. Paciente de 26 anos de idade, assintomática, com antecedente familiar de morte súbita precoce. Fonte: acervo InCor-HC-FMUSP. 
A posição de eletrodos, descrita na Figura 3, aumenta a sensibilidade diagnóstica de $\mathrm{SBr}$ e pode ser preferida em relação à posição de eletrodos-padrão para pacientes com diagnóstico suspeito ou confirmado, podendo ser realizado em pacientes com Covid-19 que apresentem parada cardíaca em TV/FV durante internação em unidade de terapia intensiva (UTI), especialmente quando associada a quadros febris. Por outro lado, para análise de outras patologias, comuns no curso da infecção, como miocardite e infarto, a escolha dos eletrodos em posição superiores deve estar bem identificada no ECG, para não induzir a erros de interpretação com relação à progressão da onda $\mathrm{R}$ e amplitude dos complexos. A medida do intervalo QT pode ser realizada na derivação D2 no ECG com derivações superiores, visto que as derivações periféricas são mantidas em posição padrão.

As principais medidas para manejo dos pacientes com SBr em UTI são prevenir a febre e evitar uso de medicações que acentuem o defeito do canal. Vários são os exemplos de medicações que aumentam risco de morte súbita nos portadores de $\mathrm{SBr}$, como fármacos de suporte no paciente internado (difenidramina), no tratamento de arritmias (amiodarona e propafenona), agentes anestésicos (propofol) e analgésicos (tramadol).

A importância da febre nos pacientes com SBr está bem estabelecida. Em geral, há aumento do intervalo PR, da duração do QRS e aumento do ponto J. A febre aumenta, inclusive, o risco de arritmias em maiores de 70 anos, quando o risco da doença já está reduzido. ${ }^{30}$ Pacientes considerados de alto risco, com temperatura acima de $38,5^{\circ} \mathrm{C}$, apesar do uso de antipiréticos, devem procurar atendimento médico. ${ }^{7}$

A SBr foi a primeira arritmia genética a ganhar destaque em publicações durante a pandemia. Foi relatado o caso de um paciente que desmascarou o padrão eletrocardiográfico durante um estado febril. Por se tratar de um paciente jovem, sem critérios de internação hospitalar, os autores optaram por dar alta com LifeVest, indisponível no Brasil. ${ }^{31}$

A hidroxicloroquina e a azitromicina podem ser indicadas, a depender da avaliação de riscos e benefícios. Sugerimos dosar eletrólitos sanguíneos e considerar monitoramento durante o tratamento. O prolongamento do intervalo QT e a dispersão da repolarização com hidroxicloroquina e azitromicina

Tabela 2 - Fenocópias de síndrome de Brugada

\begin{tabular}{l}
\hline Condições metabólicas \\
\hline Crise adrenal, acidose metabólica, hiperglicemia \\
\hline Distúrbios eletrolíticos \\
\hline Hipercalemia, hipocalemia e hiponatremia \\
\hline Compressão mecânica na via de saída do ventrículo direito \\
\hline Tumores, pectus excavatum \\
\hline Outras \\
\hline Isquemia miocárdica e embolia pulmonar \\
\hline Pericardite e miocardite \\
\hline Medicações e intoxicações exógenas \\
\hline Fonte: www.brugadadrugs.org
\end{tabular}

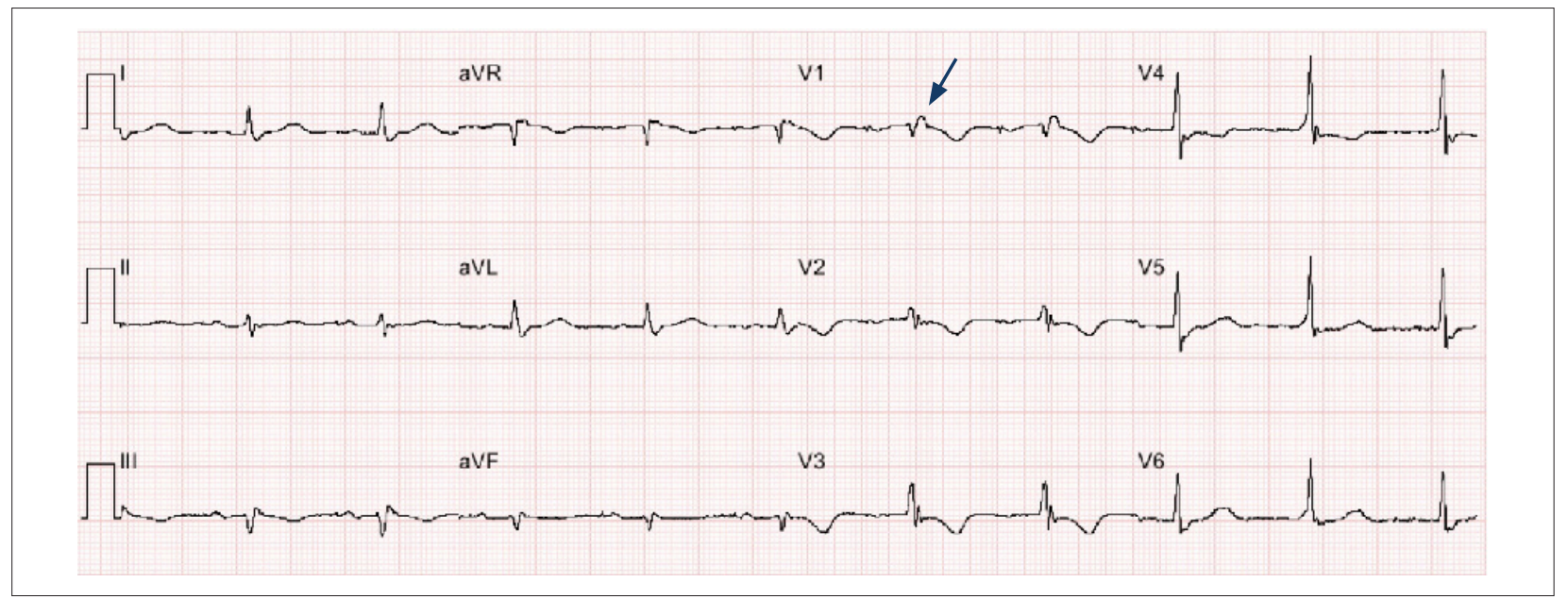

Figura 3 - Eletrocardiograma de paciente com cardiomiopatia arritmogênica de ventrículo direito. Paciente de 45 anos de idade, recuperado de parada cardíaca. Observa-se a presença de onda épsilon (seta) e onda T negativa de V1 a V3, ambos critérios maiores, além de baixa voltagem em plano frontal, denotando grave acometimento ventricular.

Fonte: acervo InCor-HC-FMUSP. 
poderiam aumentar o risco de arritmia em pacientes com $\mathrm{SBr}$, mesmo sem relação direta dessas medicações com os canais despolarizantes. ${ }^{23,32}$

O tratamento da tempestade elétrica na Sbr tem como objetivo aumentar a corrente de cálcio (despolarizante), a fim de se obter normalização do supradesnivelamento do segmento ST e reduzir a reentrada de fase 2 . O isoproterenol, como agonistas beta-adrenérgicos, pode ser eficaz, preferencialmente em associação com quinidina (indisponível no Brasil). Os inibidores da fosfodiesterase III, como o cilostazol oral ou o milrinone endovenoso, apresentaram redução de arritmogenicidade em modelos experimentais de Sbr, mas seus efeitos em humanos ainda estão em andamento. ${ }^{33}$

\section{Cardiomiopatia arritmogênica do ventrículo direito e/ou esquerdo}

\section{Aspectos gerais}

A cardiomiopatia arritmogênica do ventrículo direito e/ou esquerdo, também conhecida como displasia ou cardiomiopatia arritmogênica do ventrículo direito (CAVD/DAVD), tem uma prevalência média de 1:5000 na população geral. ${ }^{34}$ É uma doença heterogênea, com diversas apresentações clínicas que pode ter como primeira manifestação a morte súbita cardíaca, que ocorre com maior frequência durante exercício físico. Não há um exame padrão-ouro no diagnóstico de CAVD, e o escore consagrado engloba história clínica, alterações eletrocardiográficas da despolarização e repolarização (Figura 3), exames de imagem, avaliação anatomopatológica e molecular. ${ }^{35}$

Atualmente, sabe-se que a CAVD é uma condição de herança geneticamente determinada, autossômica dominante na maioria dos casos, podendo ter formas raras que são de herança autossômica recessiva, como na doença de $\operatorname{Naxos}^{36}$ ou na síndrome de Carvajal. ${ }^{37} \mathrm{~A}$ penetrância da CAVD é incompleta, com acometimento familiar em até $50 \%$ dos casos, podendo estar subestimada pela expressividade variável da doença.

A apresentação clínica da CAVD ocorre frequentemente entre a segunda e a quarta década de vida. ${ }^{38,39}$ As manifestações mais comuns são palpitações, síncope e parada cardiorrespiratória, podendo progredir para insuficiência cardíaca congestiva.

As mutações relacionadas à CAVD acometem tipicamente genes que codificam proteínas desmossomais, estruturas importantes na adesão celular do cardiomiócito que têm um papel-chave na sua fisiopatologia. Os desmossomos, além de serem estruturas especializadas em conexão celular, são importantes mediadores que atuam na transdução dos sinais intracelulares e intercelulares. ${ }^{40} \mathrm{~A}$ perda da complexa função desmossomal provoca ruptura da junção intercelular, desprendimento de miócitos e morte celular. A substituição do cardiomiócito por fibrose e gordura contribui para o desenvolvimento de área de condução lenta, que gera substrato anatômico cicatricial para macrorreentrada e arritmias ventriculares. A fibrose progride do epicárdio para o endocárdio e envolve principalmente a parede livre do VD, resultando em afilamento e dilatação aneurismática. ${ }^{41}$
O tratamento deve ser direcionado à manifestação clínica da CAVD. Não há evidência de que os antiarrítmicos previnam a morte súbita, sendo o CDI indicado em pacientes de alto risco (recuperados de PCR e TV espontânea documentada). Os betabloqueadores são considerados primeira linha no tratamento das arritmias atriais, extrassístoles ventriculares, TV não sustentada, além de importante coadjuvante no controle de choques apropriados ou inapropriados (principalmente por arritmias atriais) pelo CDI. O sotalol, a amiodarona e a ablação por radiofrequência podem ser alternativas terapêuticas, quando os betabloqueadores são ineficazes ou mal tolerados. ${ }^{42}$

\section{Cuidados na pandemia pela Covid-19}

As arritmias ventriculares em pacientes com CAVD são frequentemente desencadeadas por estresse físico e emocional, tendo um componente adrenérgico-dependente importante. Dessa forma, o aumento da liberação adrenérgica relacionado à reposta compensatória da síndrome inflamatória que acompanha o quadro infeccioso pode ser indutor das arritmias ventriculares. Deve-se manter o betabloqueador nesses pacientes enquanto houver condição hemodinâmica e, se possível, os antiarrítmicos (sotalol, amiodarona). Os fármacos com efeito alfa ou beta-adrenérgico - como aminas vasoativas (epinefrina, norepinefrina) e inotrópicos (dobutamina, milrinone) - podem aumentar o risco de arritmias ventriculares; contudo, manter a estabilidade hemodinâmica é soberana em pacientes críticos.

Estima-se que cerca de $17 \%$ dos pacientes internados pela Covid-19 necessitam de intubação orotraqueal e ventilação mecânica para recuperação. ${ }^{1}$ A ventilação mecânica tem efeitos hemodinâmicos sobre VD como aumento de pós-carga direita e redução do débito cardíaco direito em pacientes com disfunção do VD e aumento da pressão venosa central. ${ }^{43}$

Os distúrbios eletrolíticos (hipocalemia, hipocalcemia ou hipomagnesemia) podem também aumentar a suscetibilidade em pacientes com substrato anatômico, como é o caso da CAVD e, portanto, a vigilância de eletrólitos deve ser rigorosa.

A hidroxicloroquina e a azitromicina sabidamente prolongam a repolarização ventricular. Dessa maneira, sua associação com os antiarrítmicos da classe III de VaughanWilliams como sotalol e amiodarona, pode potencializar o risco de atividade deflagrada por pós-potenciais precoces, podendo causar TdP/FV. Conforme sugerido por alguns autores, a amiodarona poderia exercer ação antiviral. ${ }^{44}$ Os antivirais como ritonavir/lopinavir não apresentam efeito catecolaminérgico que aumentem o risco arrítmico em pacientes com cardiopatia e não há evidências que possam interagir com betabloqueadores/antiarrítmicos habitualmente usados na CAVD. ${ }^{45}$

\section{Taquicardia ventricular polimórfica catecolaminérgica}

\section{Aspectos gerais}

A TVPC ocorre em aproximadamente 1 em 10.000 pessoas, acomete principalmente crianças na primeira e segunda 
década de vida, com síncope ou PCR recuperada, relacionada ao exercício ou à emoção. O ECG de 12 derivações em repouso é normal. O diagnóstico é feito pelo teste de exercício, após a exclusão de doença cardíaca estrutural, preferencialmente por ressonância cardíaca.

Durante o teste de exercício, as extrassístoles ventriculares aparecem com o incremento do esforço físico, quando a FC atinge $100 \mathrm{bpm}$, progredindo para TV polimórfica e, às vezes, para TV bidirecional clássica que é considerada patognomônica dessa canalopatia (Figura 4). ${ }^{10}$

A arritmia implicada na TVPC ocorre pela perda de recaptação de cálcio no citosol. A epinefrina, liberada durante o esforço, promove acentuada liberação adicional de cálcio do retículo sarcoplasmático durante a diástole. O acionamento das correntes de sódio e cálcio leva a um acentuado influxo de sódio, que pode despolarizar a célula e provocar extrassístoles por atividade deflagrada por pós-potenciais tardios. A emergência de extrassístoles ventriculares randômicas pelo sistema de Purkinje é responsável pelo aspecto polimórfico da TV.

Aproximadamente $60 \%$ dos pacientes com TVPC apresentam defeito no receptor cardíaco de rianodina, codificado pelo gene RyR2 (TVPC tipo 1). A TVPC tipo 2 é mais rara e representa a doença em seu padrão de herança autossômico recessivo, causado por mutações da calsequestrina (CASQ2). Alguns casos de TVPC, ainda mais incomuns, foram relacionados a outras proteínas relacionadas à homeostase do cálcio, gerando o mesmo padrão de arritmias ventriculares. Os genes implicados nessas recentes descobertas foram CALM1 (que codifica a calmodulina) e TRDN (codificação da triadina). As mutações no KCNJ2 e TECRL também já foram descritas. ${ }^{10}$

O objetivo principal da terapia é o bloqueio adrenérgico com propranolol ou nadolol, que pode ser reforçado pela simpatectomia cardíaca esquerda, em pacientes que permanecem sintomáticos ou sem redução das arritmias em testes ergométricos. A flecainida (indisponível no Brasil), recentemente, mostrou benefício no tratamento por inibir a liberação de cálcio mediada pela rianodina (opção, se efeito de classe, com propafenona). ${ }^{29}$

$\mathrm{O}$ CDI deve ser indicado principalmente em recuperados de PCR. Entretanto, diferentemente de outras canalopatias, o choque pode induzir a liberação de epinefrina e morte por tempestade elétrica; portanto, a otimização farmacológica é soberana. ${ }^{46}$

\section{Cuidados na pandemia pela Covid-19}

Os pacientes com TVPC, mesmo com controle adequado de sintomas e de arritmias ventriculares, podem apresentar recorrências potencialmente fatais se houver suspensão ou redução dos betabloqueadores e, portanto, é importante manutenção das medicações intra e extra-hospitalares, ponderando o status hemodinâmico do doente mais crítico.

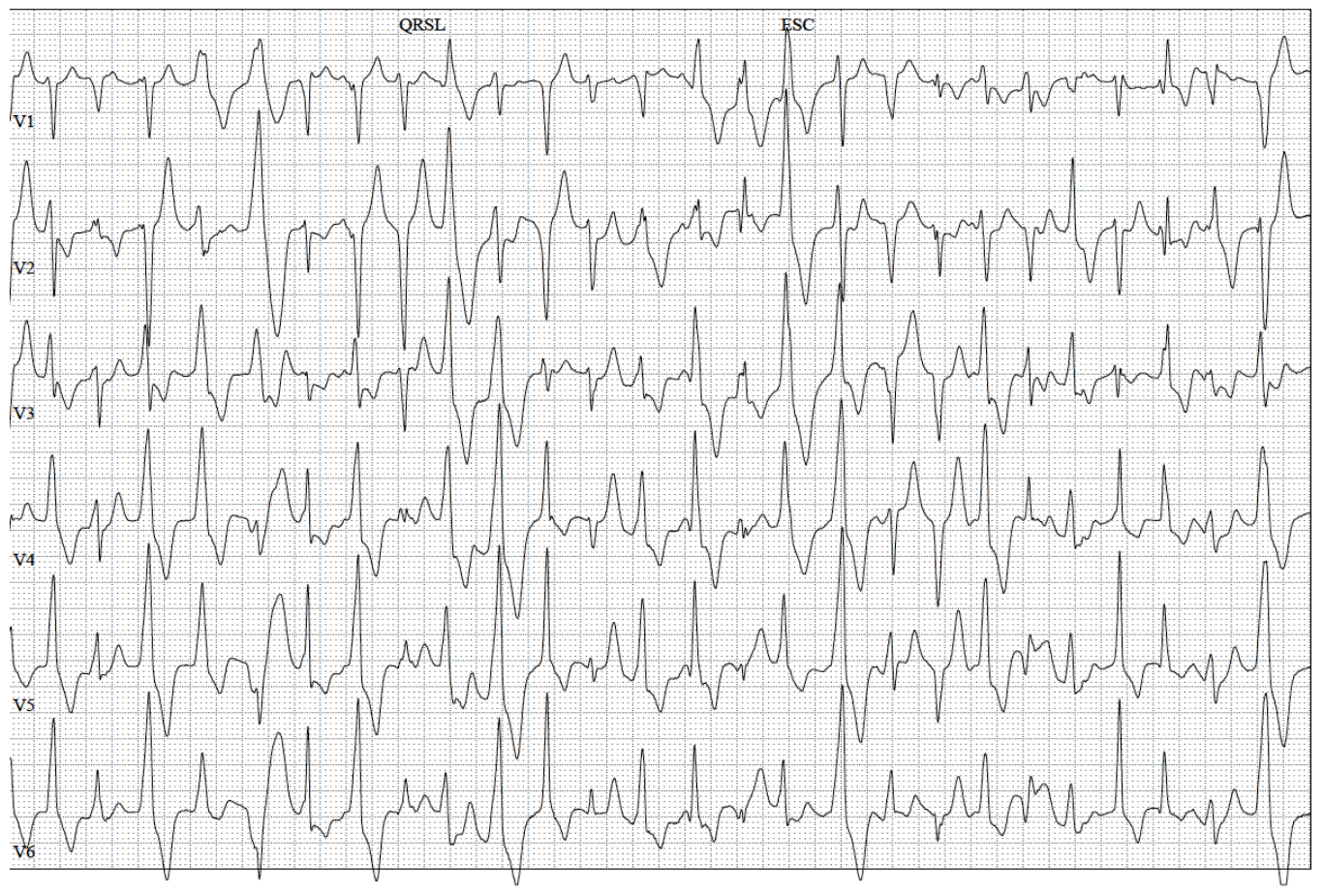

Figura 4 - Protótipo da arritmia ventricular em pacientes com taquicardia ventricular polimórfica catecolaminérgica. Paciente de 26 anos de idade, com arritmia ventricular multifocal nas primeiras etapas do esforço físico, com antecedente familiar de morte súbita na primeira década de vida. Fonte: acervo InCor-HC-FMUSP. 
Fármacos com efeito alfa ou beta-adrenérgico como aminas vasoativas (epinefrina, norepinefrina) e inotrópicos (dobutamina, milrinone) utilizados habitualmente para suporte hemodinâmico podem aumentar o risco de arritmias ventriculares em pacientes com TVPC. A epinefrina é usada para teste farmacológico na TVPC devido ao seu potencial de desmascarar as arritmias ventriculares, e, portanto, se o paciente necessitar de suporte hemodinâmico, outras aminas vasoativas devem ser preferidas à epinefrina. ${ }^{7,47}$

O milrinone, inibidor de fosfodiesterase 3, reduz a degradação do monofosfato de adenosina cíclica (AMPc), aumentando a liberação de cálcio pelo receptor de rianodina, que é a patogênese da TVPC. Em algumas situações específicas, considerando o comprometimento hemodinâmico, pode ser possível utilizar dose baixa de bloqueadores de receptores beta 1 (propranolol). ${ }^{48}$

Durante uma infecção grave, os pacientes podem não tolerar os betabloqueadores e antiarrítmicos de uso crônico e, durante todo esse período de maior vulnerabilidade arrítmica, deve-se atentar a distúrbios hidreletrolíticos e evitá-los.

Antivirais como ritonavir/lopinavir não apresentam potencial interação com betabloqueadores ou efeito catecolaminérgico que aumentem o risco arrítmico em pacientes com TVPC, porém podem interagir com a flecainida - fármaco coadjuvante no tratamento da TVPC. ${ }^{45}$ No Brasil, na falta de comercialização de flecainida, utilizamos propafenona, que também pode interagir com os antivirais.

A hidroxicloroquina, aparentemente, não aumenta os níveis de catecolaminas. No entanto, há evidências de interação medicamentosa entre a hidroxicloroquina e o propranolol/nadolol. Os betabloqueadores são metabolizados via citocromo CYP2D6, e sua inibição pela hidroxicloroquina pode acarretar aumento da concentração do medicamento, exigindo monitoramento cuidadoso da frequência cardíaca e da pressão arterial. ${ }^{49} \mathrm{~A}$ flecainida e a propafenona apresentam uma interação semelhante, resultando em aumento do nível

\section{Referências}

1. Zhou F, Yu T, Du R, Fan G, Liu Y, Liu Z, et al. Clinical Course and Risk Factors for Mortality of Adult Inpatients with COVID-19 in Wuhan, China: A Retrospective Cohort Study. Lancet. 2020;395(10229):105462. doi: 10.1016/S0140-6736(20)30566-3.

2. People with Certain Medical Conditions [Internet]. Washington: Centers for Disease Control and Prevention; 2021 [cited 2021 Jul 12]. Available from: https://www.cdc.gov/coronavirus/2019-ncov/needextra-precautions/people-with-medical-conditions.html.

3. Wunderink RG, Waterer G. Advances in the Causes and Management of Community Acquired Pneumonia in Adults. BMJ. 2017;358:j2471. doi: 10.1136/bmj.j2471.

4. Corrales-Medina VF, Musher DM, Shachkina S, Chirinos JA. Acute Pneumonia and the Cardiovascular System. Lancet. 2013;381(9865):496-505. doi: 10.1016/S0140-6736(12)61266-5.

5. Marrie TJ, Shariatzadeh MR. Community-Acquired Pneumonia Requiring Admission to an Intensive Care Unit: A Descriptive Study. Medicine. 2007;86(2):103-11. doi: 10.1097/MD.0b013e3180421c16.

6. Carr GE, Yuen TC, McConville JF, Kress JP, VandenHoek TL, Hall JB, et al. Early Cardiac Arrest in Patients Hospitalized With Pneumonia: A Report sérico dos antiarrítmicos, potencializando risco arrítmico. ${ }^{45}$ Nessas situações, devemos pesar risco e benefício individual para decisão terapêutica.

\section{Conclusão}

Os pacientes com arritmias hereditárias apresentam diversos fatores moleculares e estruturais que os predispõem a eventos potencialmente fatais no curso de uma infecção viral. A pandemia pela Covid-19 nos leva a distanciá-los do risco de infecção, reforçando medidas de isolamento e higiene, além de orientar precauções nos cuidados médicos, relembrando as peculiaridades de portadores de doenças raras. Dentre as recomendações, reforçamos os cuidados com relação às medicações utilizadas pelo paciente, ao tratamento efetivo da febre e dos distúrbios eletrolíticos e ao risco de prescrição de medicações com potencial pró-arrítmico.

\section{Contribuição dos autores}

Concepção e desenho da pesquisa: todos os autores. Redação do manuscrito: Sacilotto L, Olivetti N. Revisão crítica do manuscrito quanto ao conteúdo intelectual importante: Pisani C, Hachul D, Darrieux F, Scanavacca MI.

\section{Potencial Conflito de Interesse}

Declaro não haver conflito de interesses pertinentes.

\section{Fontes de Financiamento}

O presente estudo não teve fontes de financiamento externas.

\section{Vinculação Acadêmica}

Não há vinculação deste estudo a programas de pósgraduação.
From the American Heart Association's Get With The GuidelinesResuscitation Program. Chest. 2012;141(6):1528-36. doi: 10.1378/ chest.11-1547.

7. Wu Cl, Postema PG, Arbelo E, Behr ER, Bezzina CR, Napolitano C, et al. SARS-CoV-2, COVID-19, and Inherited Arrhythmia Syndromes. Heart Rhythm. 2020;17(9):1456-62. doi: 10.1016/j.hrthm.2020.03.024.

8. Giudicessi JR, Ackerman MJ. Determinants of Incomplete Penetrance and Variable Expressivity in Heritable Cardiac Arrhythmia Syndromes. TransI Res. 2013;161(1):1-14. doi: 10.1016/j.trsl.2012.08.005.

9. Qiu J, Shen B, Zhao M, Wang Z, Xie B, Xu Y. A Nationwide Survey of Psychological Distress Among Chinese People in the COVID-19 Epidemic: Implications and Policy Recommendations. Gen Psychiatr. 2020;33(2):e100213. doi: 10.1136/gpsych-2020-100213.

10. Skinner JR, Winbo A, Abrams D, Vohra J, Wilde AA. Channelopathies That Lead to Sudden Cardiac Death: Clinical and Genetic Aspects. Heart Lung Circ. 2019;28(1):22-30. doi: 10.1016/j.hlc.2018.09.007.

11. Bohnen MS, Peng G, Robey SH, Terrenoire C, lyer V, Sampson KJ, et al. Molecular Pathophysiology of Congenital Long QT Syndrome. Physiol Rev. 2017;97(1):89-134. doi: 10.1152/physrev.00008.2016. 
12. Wu TC, Sacilotto L, Darrieux FCDC, Pisani CF, Melo SL, Hachul DT, et al. QT Interval Control to Prevent Torsades de Pointes during Use of Hydroxychloroquine and/or Azithromycin in Patients with COVID-19. Arq Bras Cardiol. 2020;114(6):1061-6. doi: 10.36660/abc.20200389.

13. Priori SG, Blomström-Lundqvist C, Mazzanti A, Blom N, Borggrefe $M$, Camm J, et al. 2015 ESC Guidelines for the Management of Patients with Ventricular Arrhythmias and the Prevention of Sudden Cardiac Death: The Task Force for the Management of Patients with Ventricular Arrhythmias and the Prevention of Sudden Cardiac Death of the European Society of Cardiology (ESC). Endorsed by: Association for European Paediatric and Congenital Cardiology (AEPC). Eur Heart J. 2015;36(41):2793-867. doi: 10.1093/eurheartj/ehv316.

14. Al-Khatib SM, Stevenson WG, Ackerman MJ, Bryant WJ, Callans DJ, Curtis AB, et al. 2017 AHA/ACC/HRS Guideline for Management of Patients with Ventricular Arrhythmias and the Prevention of Sudden Cardiac Death: Executive Summary: A Report of the American College of Cardiology/ American Heart Association Task Force on Clinical Practice Guidelines and the Heart Rhythm Society. Heart Rhythm. 2018;15(10):190-252. doi: 10.1016/j.hrthm.2017.10.035

15. Amin AS, Herfst LJ, Delisle BP, Klemens CA, Rook MB, Bezzina CR, et al. Fever-Induced QTC Prolongation and Ventricular Arrhythmias in Individuals with type 2 Congenital Long QT Syndrome. J Clin Invest. 2008;118(7):255261. doi: 10.1172/JCI35337.

16. Salinas P, Lopez-de-Sa E, Pena-Conde L, Viana-Tejedor A, Rey-Blas JR, Armada E, et al. Electrocardiographic Changes During Induced Therapeutic Hypothermia in Comatose Survivors After Cardiac Arrest. World J Cardiol. 2015;7(7):423-30. doi: 10.4330/wjc.v7.i7.423.

17. El-Sherif N, Turitto G, Boutjdir M. Acquired Long QT Syndrome and Torsade de Pointes. Pacing Clin Electrophysiol. 2018;41(4):414-21. doi: 10.1111/ pace.13296.

18. Lazzerini PE, Capecchi PL, Laghi-Pasini F, Boutjdir M. Autoimmune Channelopathies as a Novel Mechanism in Cardiac Arrhythmias. Nat Rev Cardiol. 2017;14(9):521-35. doi: 10.1038/nrcardio.2017.61.

19. Giudicessi JR, Ackerman MJ, Camilleri M. Cardiovascular Safety of Prokinetic Agents: A Focus on Drug-Induced Arrhythmias. Neurogastroenterol Motil. 2018;30(6):e13302. doi: 10.1111/nmo.13302.

20. O'Hare M, Maldonado Y, Munro J, Ackerman MJ, Ramakrishna H, Sorajja D. Perioperative Management of Patients with Congenital or Acquired Disorders of the QT Interval. Br J Anaesth. 2018;120(4):629-44. doi: 10.1016/j.bja.2017.12.040.

21. Yao X, Ye F, Zhang M, Cui C, Huang B, Niu P, et al. In Vitro Antiviral Activity and Projection of Optimized Dosing Design of Hydroxychloroquine for the Treatment of Severe Acute Respiratory Syndrome Coronavirus 2 (SARSCoV-2). Clin Infect Dis. 2020;71(15):732-9. doi: 10.1093/cid/ciaa237.

22. Mazzanti A, Briani M, Kukavica D, Bulian F, Marelli S, Trancuccio A, et al. Association of Hydroxychloroquine With QTC Interval in Patients with COVID-19. Circulation. 2020;142(5):513-5. doi: 10.1161/ CIRCULATIONAHA.120.048476.

23. Teixeira RA, Borba EF, Pedrosa A, Nishioka S, Viana VS, Ramires JA, et al. Evidence for Cardiac Safety and Antiarrhythmic Potential of Chloroquine in Systemic Lupus Erythematosus. Europace. 2014;16(6):887-92. doi: 10.1093/europace/eut290.

24. Viskin S. Long QT Syndromes and Torsade de Pointes. Lancet. 1999;354(9190):1625-33. doi: 10.1016/S0140-6736(99)02107-8.

25. Morrison LJ, Deakin CD, Morley PT, Callaway CW, Kerber RE, Kronick SL, et al. Part 8: Advanced Life Support: 2010 International Consensus on Cardiopulmonary Resuscitation and Emergency Cardiovascular Care Science With Treatment Recommendations. Circulation. 2010;122(16 Suppl 2):345-421. doi: 10.1161/CIRCULATIONAHA.110.971051.

26. Kawada S, Morita H, Antzelevitch C, Morimoto Y, Nakagawa K, Watanabe A, et al. Shanghai Score System for Diagnosis of Brugada Syndrome:
Validation of the Score System and System and Reclassification of the Patients. JACC Clin Electrophysiol. 2018;4(6):724-30. doi: 10.1016/j. jacep.2018.02.009

27. Oliveira Neto NR, Oliveira WS, Mastrocola F, Sacilotto L. Brugada Phenocopy: Mechanisms, Diagnosis, and Implications. J Electrocardiol. 2019;55:45-50. doi: 10.1016/j.jelectrocard.2019.04.017.

28. Brugada J, Campuzano O, Arbelo E, Sarquella-Brugada G, Brugada R. Present Status of Brugada Syndrome: JACC State-of-the-Art Review. J Am Coll Cardiol. 2018;72(9):1046-59. doi: 10.1016/j.jacc.2018.06.037.

29. Al-Khatib SM, Stevenson WG, Ackerman MJ, Bryant WJ, Callans DJ, Curtis AB, et al. $2017 \mathrm{AHA} / \mathrm{ACC} / \mathrm{HRS}$ guideline for management of patients with ventricular arrhythmias and the prevention of sudden cardiac death: Executive summary: A Report of the American College of Cardiology/ American Heart Association Task Force on Clinical Practice Guidelines and the Heart Rhythm Society. Heart Rhythm. 2018;15(10):e190-e252. doi: 10.1016/j.hrthm.2017.10.035

30. Michowitz Y, Milman A, Sarquella-Brugada G, Andorin A, Champagne J, Postema PG, et al. Fever-Related Arrhythmic Events in the Multicenter Survey on Arrhythmic Events in Brugada Syndrome. Heart Rhythm. 2018;15(9):1394-401. doi: 10.1016/j.hrthm.2018.04.007.

31. Chang D, Saleh M, Garcia-Bengo Y, Choi E, Epstein L, Willner J. COVID-19 Infection Unmasking Brugada Syndrome. HeartRhythm Case Rep. 2020;6(5):237-40. doi: 10.1016/j.hrcr.2020.03.012.

32. White NJ. Cardiotoxicity of Antimalarial Drugs. Lancet Infect Dis. 2007:7(8):549-58. doi: 10.1016/S1473-3099(07)70187-1.

33. Szél T, Koncz I, Antzelevitch C. Cellular Mechanisms Underlying the Effects of Milrinone and Cilostazol to Suppress Arrhythmogenesis Associated with Brugada syndrome. Heart Rhythm. 2013;10(11):1720-7. doi: 10.1016/j. hrthm.2013.07.047.

34. Peters S, Trümmel M, Meyners W. Prevalence of Right Ventricular Dysplasia-Cardiomyopathy in a Non-Referral Hospital. Int I Cardiol. 2004;97(3):499-501. doi: 10.1016/j.ijcard.2003.10.037.

35. Towbin JA, McKenna WJ, Abrams DJ, Ackerman MJ, Calkins H, Darrieux FCC, et al. 2019 HRS Expert Consensus Statement on Evaluation, Risk Stratification, and Management of Arrhythmogenic Cardiomyopathy: Executive Summary. Heart Rhythm. 2019;16(11):373-407. doi: 10.1016/j. hrthm.2019.09.019.

36. McKoy G, Protonotarios N, Crosby A, Tsatsopoulou A, Anastasakis A, Coonar A, et al. Identification of a Deletion in Plakoglobin in Arrhythmogenic Right Ventricular Cardiomyopathy with Palmoplantar Keratoderma and Woolly Hair (Naxos disease). Lancet. 2000;355(9221):2119-24. doi: 10.1016/ S0140-6736(00)02379-5.

37. Norgett EE, Hatsell SJ, Carvajal-Huerta L, Cabezas JC, Common J, Purkis $\mathrm{PE}$, et al. Recessive Mutation in Desmoplakin Disrupts DesmoplakinIntermediate Filament Interactions and Causes Dilated Cardiomyopathy, Woolly Hair and Keratoderma. Hum Mol Genet. 2000;9(18):2761-6. doi 10.1093/hmg/9.18.2761.

38. Bhonsale A, Groeneweg JA, James CA, Dooijes D, Tichnell C, Jongbloed JD, et al. Impact of Genotype on Clinical Course in Arrhythmogenic Right Ventricular Dysplasia/Cardiomyopathy-Associated Mutation Carriers. Eur Heart J. 2015;36(14):847-55. doi: 10.1093/eurheartj/ehu509.

39. Groeneweg JA, Bhonsale A, James CA, Riele AS, Dooijes D, Tichnell C, et al. Clinical Presentation, Long-Term Follow-Up, and Outcomes of 1001 Arrhythmogenic Right Ventricular Dysplasia/Cardiomyopathy Patients and Family Members. Circ Cardiovasc Genet. 2015;8(3):437-46. doi: 10.1161/ CIRCGENETICS.114.001003.

40. Basso C, Czarnowska E, Barbera MD, Bauce B, Beffagna G, Wlodarska EK, et al. Ultrastructural Evidence of Intercalated Disc Remodelling in Arrhythmogenic Right Ventricular Cardiomyopathy: An Electron Microscopy Investigation on Endomyocardial Biopsies. Eur Heart J. 2006;27(15):1847-54. doi: 10.1093/eurheartj/ehl095. 


\section{Artigo de Revisão}

41. Fontaine G, Frank R, Tonet JL, Guiraudon G, Cabrol C, Chomette G, et al. Arrhythmogenic Right Ventricular Dysplasia: A Clinical Model for the Study of Chronic Ventricular Tachycardia. Jpn Circ J. 1984;48(6):515-38. doi: 10.1253/jcj.48.515.

42. Corrado D, Wichter T, Link MS, Hauer R, Marchlinski F, Anastasakis A, et al. Treatment of Arrhythmogenic Right Ventricular Cardiomyopathy/ Dysplasia: An International Task Force Consensus Statement. Eur Heart J. 2015;36(46):3227-37. doi: 10.1093/eurheartj/ehv162.

43. Wang XT, Liu DW, Zhang HM, Long Y, Guan XD, Qiu HB, et al. Experts consensus on the management of the right heart function in critically ill patients. Zhonghua Nei Ke Za Zhi. 2017;56(12):962-73. doi: 10.3760/cm a.j.issn.0578-1426.2017.12.017.

44. Aimo A, Baritussio A, Emdin M, Tascini C. Amiodarone as a Possible Therapy for Coronavirus Infection. Eur J Prev Cardiol. 2020:2047487320919233. doi: $10.1177 / 2047487320919233$.

45. Giudicessi JR, Noseworthy PA, Friedman PA, Ackerman MJ. Urgent Guidance for Navigating and Circumventing the QTc-Prolonging and Torsadogenic Potential of Possible Pharmacotherapies for Coronavirus Disease 19 (COVID-19). Mayo Clin Proc. 2020;95(6):1213-21. doi: 10.1016/j. mayocp.2020.03.024.
46. Marai I, Khoury A, Suleiman M, Gepstein L, Blich M, Lorber A, etal. Importance of Ventricular Tachycardia Storms Not Terminated by Implantable Cardioverter Defibrillators Shocks in Patients with CASQ2 Associated Catecholaminergic Polymorphic Ventricular Tachycardia. Am J Cardiol. 2012;110(1):72-6. doi: 10.1016/j.amjcard.2012.02.049.

47. Marjamaa A, Hiippala A, Arrhenius B, Lahtinen AM, Kontula K, Toivonen L, et al. Intravenous Epinephrine Infusion Test in Diagnosis of Catecholaminergic Polymorphic Ventricular Tachycardia. J Cardiovasc Electrophysiol. 2012;23(2):194-9. doi: 10.1111/j.1540-8167.2011.02188.x.

48. Kobayashi S, Susa T, Ishiguchi H, Myoren T, Murakami W, Kato T, et al. A Low-Dose $\beta 1$-Blocker in Combination with Milrinone Improves Intracellular $\mathrm{Ca} 2+$ Handling in Failing Cardiomyocytes by Inhibition of Milrinone-Induced Diastolic Ca2 + Leakage from the Sarcoplasmic Reticulum. PLoS One. 2015;10(1):e0114314. doi: 10.1371/journal.pone.0114314.

49. Driggin E, Madhavan MV, Bikdeli B, Chuich T, Laracy J, Biondi-Zoccai G, et al. Cardiovascular Considerations for Patients, Health Care Workers, and Health Systems During the COVID-19 Pandemic.J Am Coll Cardiol. 2020;75(18):235271. doi: 10.1016/j.jacc.2020.03.031.

50. brugadadrugs.org [Internet]. Amsterdã: Amsterdam University Medical Centers; 2021 [cited 2021 Jul 12]. Available from: https://www.brugadadrugs.org/ 